\title{
Hausdorff Distance Applied On Real Data Experiment For Underwater Localization
}

\author{
Pedro Eugênio Martins de Magalhães \\ Univ. Grenoble Alpes, CNRS, G-INP, \\ GIPSA-Lab, France \\ Jérôme Mars \\ Univ. Grenoble Alpes, CNRS, G-INP, \\ GIPSA-Lab, France
}

\author{
Cornel Ioana \\ Univ. Grenoble Alpes, CNRS, G-INP, \\ GIPSA-Lab, France \\ Xavier Cristol \\ Thales Underwater Systems \\ Sophia Antipolis, France
}

\begin{abstract}
This paper addresses the problem of localizing and tracking a surface or underwater vessel with the technique called as Hausdorff Distance. Two proposed approaches, based on TDOAs comparison, were used for 2-D localization, in range and depth, with one sensor only, and have been successfully applied to localize a motionless unknown target in a tank's experiment. Results in terms of the localization accuracy have been obtained with real signal and the performance of the proposed localization techniques have been demonstrated and confirmed by simulation with respect of signal-to-noise ratio and compared with the correlation techniques used nowadays for single hydrophones.
\end{abstract}

Keywords-Underwater source localization; Underwater Ray path propagation; Hausdorff Distance; TDOAs correlation; Geoacoustic Inversion Underwater problem. Matched Field Processing.

\section{INTRODUCTION}

The main goal of this work is to build a robust underwater localization program, despite inaccurate sound speed profile and/or bottom depths, and ocean noise information. Through this innovative matching Hausdorff technique, used for underwater localization, it becomes possible localize the target in a 2-D scenario, range and depth, with only one sensor at the receiver, and being more accurate and faster that previous techniques, and insensible to variation in phase.

Localization of acoustic sources is usually done by using the measurement of the time difference of arrival (TDOA) [1]. Nowadays several solutions exist for localization called as matched field processing, based on cross correlation processing [2], the Bartlett estimator [3] and high resolution Algorithms (adaptive beamforming) such as: Maximum Likehood [4], MUSIC with real data experiment [5], ESPRIT [6]. However all those techniques are common for an array of hydrophones which have the advantage of diversity.

The Hausdorff Distance technique is commonly used in many domains, generally in image processing and pattern matching, such as: Matching radar and satellite images [7], ship positioning by matching radar images with satellite images [8], speech recognition, [9], video recognition, [10], underwater vehicles classification, [11] [12], sidescan sonar [13], and others [14]. In this paper we are interesting on applying for the first time a direct comparison only in time domain using the time difference of arrival (TDOA), obtained from the experiment and compared with the simulated from a ray path program developed by the authors.

This paper is organized as follow. Section II aims to present an underwater propagation program based on ray propagation theory, equivalent to Bellhop, used to obtain the TDOAs references. Section III we present the localization techniques used to find the best match between the TDOA emulated from the propagation program and the TDOA recorded at the experiment. In section IV we explain the experiment set-up recorded at the GISPA-Lab tank in Grenoble, France. Section $\mathrm{V}$, we present the simulations in order to check the accuracy of each technique with respect to signal noise ratio (SNR). In Section VI, the main results of the paper are summarized in term of precision at the experiment. Finally, Section VII, we present the conclusion of this paper.

\section{UNDERWATER PROPAGATION}

\section{A. Ray Propagation Theory}

Ray Path propagation has been used since early 1960s, commonly for high frequencies and deep water [1]. The ray propagation theory is mainly described in [2] [3]. In this work, we are interested in how to obtain the TDOA from the ray propagation theory to the localization problem. We want to understand its variation with the depth and range, which in some scenarios may works as a fingerprint being only associated with a unique point in space.

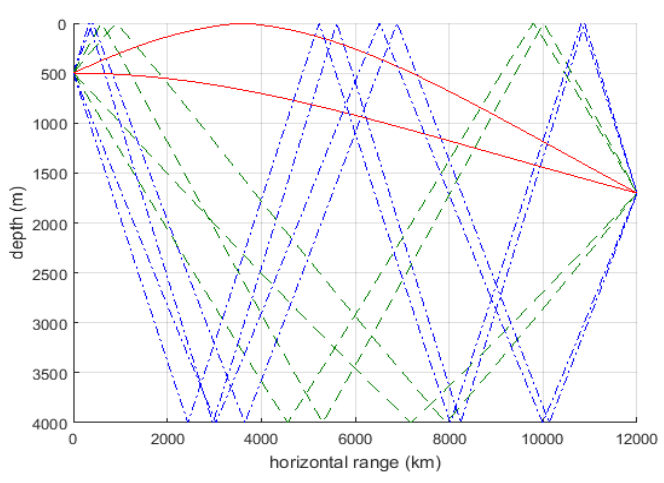

Fig. 1. Rays Paths pack for a certain position in space. 
In a deep ocean scenario, sound may propagate along different paths, shown at "Fig 1". Each ray path suffers small variations of its curvature due to variation of the sound speed profile, according to Snell's law. In our plot, we consider a canonic Munk's sound speed profile and a range independent scenario. The received signal is composed of several arrivals that are the time shifted and attenuated versions of the emitted signal, originating from, direct path, surface reflection, bottom reflection, and surface duct. In our work, we only consider 3 different groups, which differ between themselves by the number of the bottom reflections.

On the first group, zero bottom reflection, we have 2 rays called as direct path and one surface (S) reflection, solid line at "Fig 1", second group, once bottom reflection, we count 4 rays, called as bottom (B), BS, SB, SBS reflections, dashed line, and at third group, twice bottom reflection, 4 rays, BSB, BSBS, SBSB, SBSBS reflections, dash-dot line. We also defined to maximum of 2 bottom reflections, due to high attenuation, around $20 \mathrm{~dB}$, that lead the signal's level to be too weak and in some cases below the level of noise.

The localization procedure, "Fig 2", consist of: Simulate a 2D scenario (range and depth), and record a target in any position on the grid of the simulation. In our experiment we used a step of $0.1 \mathrm{~m}$ either for range and depths. The next step consists of finding the best match between simulated and recorded, using the techniques proposed in this work, which should be close to the real target position. The set of all delays and attenuation together with the channel environmental conditions provides sufficient information for determining the source location, however in some situation it can also give us ambiguity results. The accuracy will be improved once that the input parameters on the simulation are as closer as possible to the real environment.

\section{LOCALIZATION TECHNIQUES}

\section{A. Maximum Hausdorff Distance}

The comparison of TDOA was done by using the Hausdorff distance (HD), proposed by Huttenlocher [15], which is a technique to measure the degree of similarity among different objects, giving an interesting measure of their mutual proximity. The matching is performed through the minimization of the algorithm between those two sets of point.

Let consider two sets of points, one being the received TDOA $R_{\mathrm{T}}=\left\{\mathrm{r}_{1}, \mathrm{r}_{2}, \mathrm{r}_{3}, \ldots \mathrm{r}_{\mathrm{x}}\right\}$, and the other the simulated TDOA $\mathrm{S}_{\mathrm{T}}=\left\{\mathrm{s}_{1}, \mathrm{~s}_{2}, \mathrm{~s}_{3}, \ldots \mathrm{s}_{\mathrm{y}}\right\}$, the HD between them is small when every point in one of the sets is near to some point in the other, been defined as:

$$
\mathrm{H}\left(\mathrm{R}_{\mathrm{T}}, \mathrm{S}_{\mathrm{T}}\right)=\max \left\{\mathrm{h}\left(\mathrm{R}_{\mathrm{T}}, \mathrm{S}_{\mathrm{T}}\right), \mathrm{h}\left(\mathrm{S}_{\mathrm{T}}, \mathrm{R}_{\mathrm{T}}\right)\right\}
$$

Where:

$$
\begin{aligned}
& \mathrm{h}\left(\mathrm{R}_{\mathrm{T}}, \mathrm{S}_{\mathrm{T}}\right)=\max _{\mathrm{r} \in \mathrm{R}}\left[\min \left(\left\|r_{i}-s\right\|\right)\right] \quad \mathrm{i}=\{1,2,3, \ldots, \mathrm{x}\} \\
& \mathrm{h}\left(\mathrm{S}_{\mathrm{T}}, \mathrm{R}_{\mathrm{T}}\right)=\max _{\mathrm{s} \in \mathrm{S}}\left[\min \left(\left\|s_{i}-r\right\|\right)\right] \quad \mathrm{j}=\{1,2,3, \ldots, \mathrm{y}\}
\end{aligned}
$$

The Euclidean distance function was used to evaluate the norm $\|\cdot\|$, and selects the farthest which is the largest discrepancy between the two sets of points or maximal distance.

\section{B. Mean Hausdorff Distance}

A variation of the technique was done by Dubuisson, in 1994, called Modified Hausdorff Distance (MHD) with the Mean Hausdorff Distance [16]. The main difference is that all points contribute to measure the average of the distance, which ensures that more measurement points closely resemble the model. In other words this can soften the problems with false alarm or fake point at the received. It is given by:

$$
\begin{aligned}
& \overline{\mathrm{h}}\left(\mathrm{R}_{\mathrm{T}}, \mathrm{S}_{\mathrm{T}}\right)=\frac{1}{\mathrm{X}} \sum_{\mathrm{i}=1}^{\mathrm{X}}\left[\min _{r \in R}\left(\left\|r_{i}-s\right\|\right)\right] \quad \mathrm{i}=\{1,2,3, \ldots, \mathrm{x}\} \\
& \overline{\mathrm{h}}\left(\mathrm{S}_{\mathrm{T}}, \mathrm{R}_{\mathrm{T}}\right)=\frac{1}{\mathrm{Y}} \sum_{\mathrm{j}=1}^{\mathrm{Y}}\left[\min _{s \in S}\left(\left\|s_{i}-r\right\|\right)\right] \quad \mathrm{j}=\{1,2,3, \ldots, \mathrm{y}\}
\end{aligned}
$$

In this work we only consider one side, $h\left(R_{T}, S_{T}\right)$ and $\overline{\mathrm{h}}\left(\mathrm{R}_{\mathrm{T}}, \mathrm{S}_{\mathrm{T}}\right)$, which is the recorded, used as reference, into the

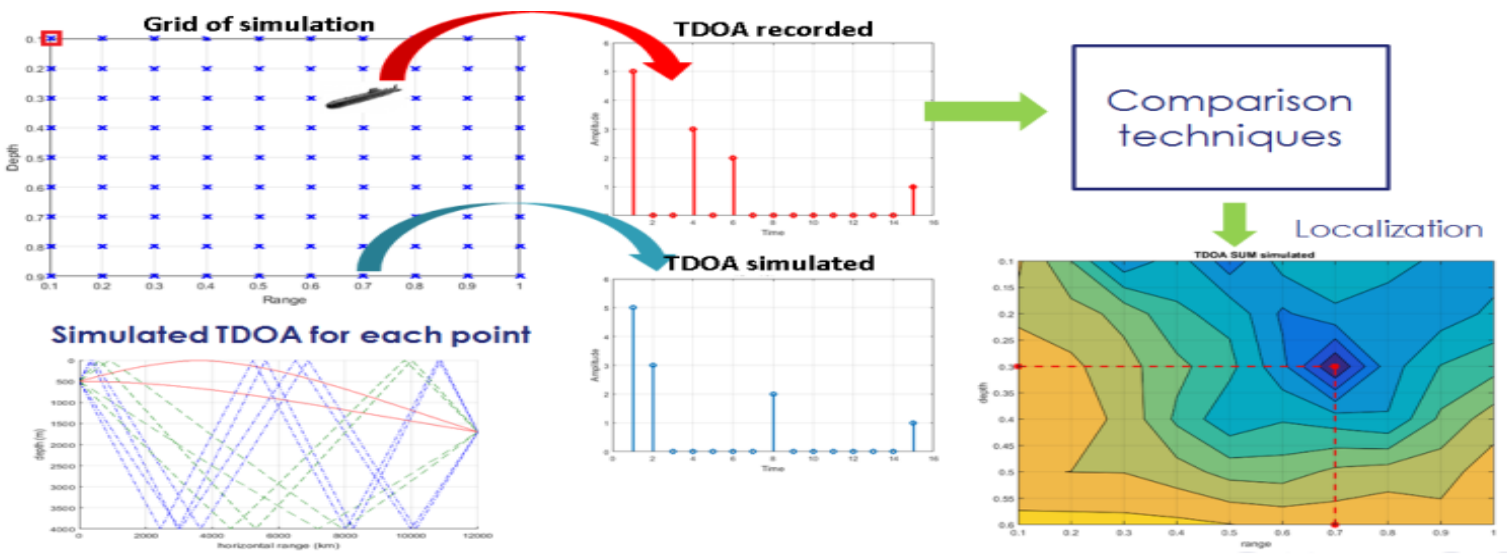

Fig. 2. Schematization of the localization problem. 
simulated, because in a long distance the signal of the last path, 2 bottom reflection, will not be detect anymore, not being possible to compare the last paths with the simulated one, which in this situation it can generate a mismatch of the localization.

\section{Correlation}

In 1998, Porter [17] has shown a technique that is applied in our problem, considering only one sensor at the receiver, and it is recommended for a known signal. Using the time domain he computed the correlation in time domain, however this technique is sensible to phase changes, which occurs with the bottom interaction. To solve this problem Porter only consider the envelope of the signal.

$$
\begin{aligned}
& \mathrm{S}_{(\mathrm{r}, \mathrm{d})}(\mathrm{t})=x(\mathrm{t}) * \mathrm{~h}_{(\mathrm{r}, \mathrm{d})}\left(\mathrm{t}, \theta_{\mathrm{s}}\right)+\mathrm{n} \\
& \mathrm{h}_{(\mathrm{r}, \mathrm{d})}\left(\mathrm{t}, \theta_{\mathrm{s}}\right)=\sum_{x=1}^{X} a_{x}\left(\theta_{\mathrm{s}}\right) \delta\left[t-\tau_{x(\mathrm{r}, \mathrm{d})}\left(\theta_{\mathrm{s}}\right)\right]
\end{aligned}
$$

Where:

$\mathrm{S}_{(\mathrm{r}, \mathrm{d})}(\mathrm{t})$ : is the simulated signal for each variation in range and in depths.

$x(\mathrm{t}):$ is the transmitted signal.

$\mathrm{h}_{(\mathrm{r}, \mathrm{d})}\left(\mathrm{t}, \theta_{\mathrm{s}}\right):$ is the impulse response for each position.

$a_{x}\left(\theta_{\mathrm{s}}\right):$ is the amplitude for each different take off angle.

Later it is applied the correlation with the recorded signal $R(t)$, and the best result, which most resemble the real position is taken by the maximum value.

$$
X \operatorname{corr}_{(\mathrm{r}, \mathrm{d})}=\max \left|\int R(\tau) \mathrm{S}_{(\mathrm{r}, \mathrm{d})}(\tau-\mathrm{t}) \mathrm{d} \tau\right|
$$

\section{EXPERIMENT IN THE TANK (GIPSA-LAB )}

In order to test and prove the applicability of the Hausdroff Distance for underwater localization problems, we conducted one experiment in the tank of the laboratory of GIPSA-Lab at University of Grenoble - France, whose size is 1.5 meters' length by 1 meter width by 1 meter height. The main goal is validate this new technique with respect of the precision of the real position. The measurement setup consists of a 2 hydrophones, one at the receiver and one at the transmitter. The sensors have 7 degrees' beam spread at $1 \mathrm{MHz}$ for 0.5 -inch diameter, which allows us to disregard the tank's side considering a narrow beam spread. The transmitted signal was a chirp with time duration of $100 \mu \mathrm{s}$ and frequency range of $500 \mathrm{KHz}$ to $1.5 \mathrm{MHz}$. The signal was sampled at $25 \mathrm{MHz}$ with a resolution of 16 bits. The medium was considered as homogeneous, used for approximation of the linear wave equation, also with a flat bottom and a constant SSP. The target was motionless so the Doppler Effect was disregarded. Due to the directivity of the sensor we have to mechanically tilt the sensor to obtain the entire set of different take off angles, reason to we accept $10 \%$ of error in depth position $(9 \mathrm{~cm})$. We set the position of the receiver at $0.1 \mathrm{~m}$ deep and the transmitter was located at $0.3 \mathrm{~m}$ deep and $0.7 \mathrm{~m}$ horizontally range from the receiver, centered horizontally in the tank as shown at "Fig $3 "$.

The record procedure consists of align the sensors for each different take-off angle, recording each ray path separately, "Fig 4", and the sum in time all different paths, using the transmitted signal as reference in time for synchronization of the different recorded signals, "Fig 5". Once that we have all package containing 9 different take-off angles, called for complete received signal, we apply the auto-correlation, and after, selecting the peaks that will be used to compare with the simulated TDOA, using the Hausdorff techniques, in order to find the correct position. In our case, the 2 amplifiers, one for $\mathrm{Tx}$ and another for $\mathrm{Rx}$, were not kept constant, since that in our first experiment the most important was to have the information of the time from each ray path. The signal noise ratio for this measure was $23.69 \mathrm{~dB}$.

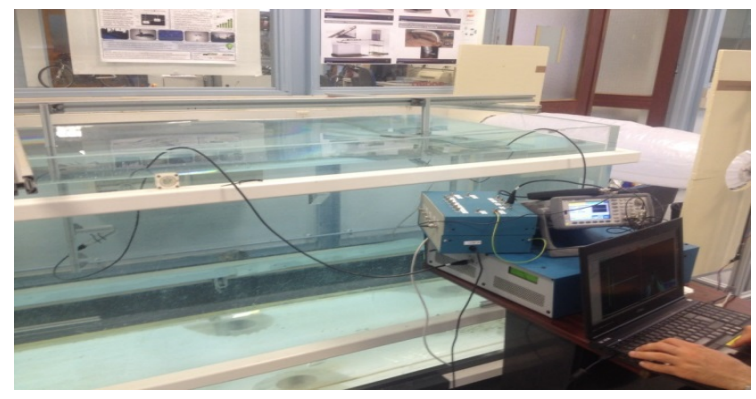

Fig. 3. Experimental facility.

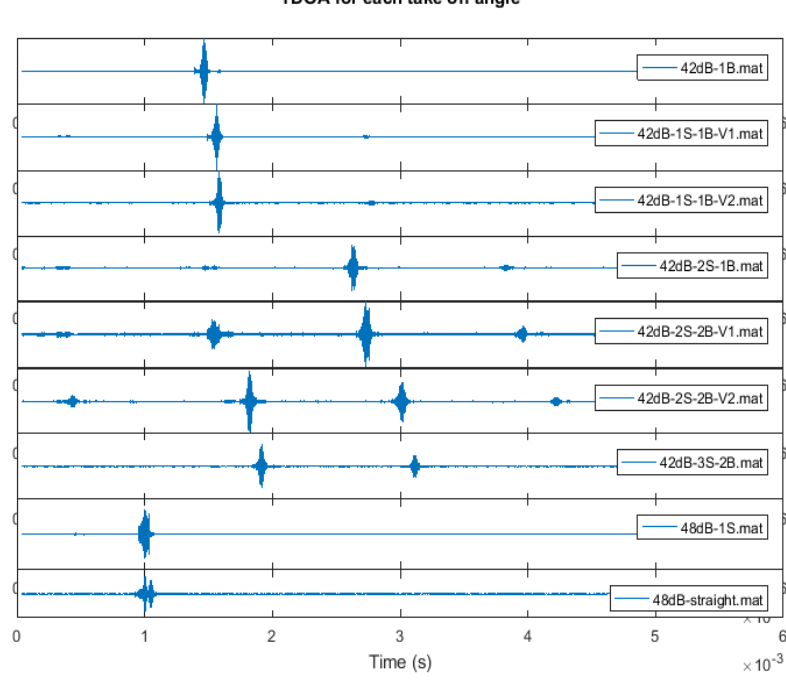

Fig. 4. TDOA recorded of 9 diferent take-off angles.

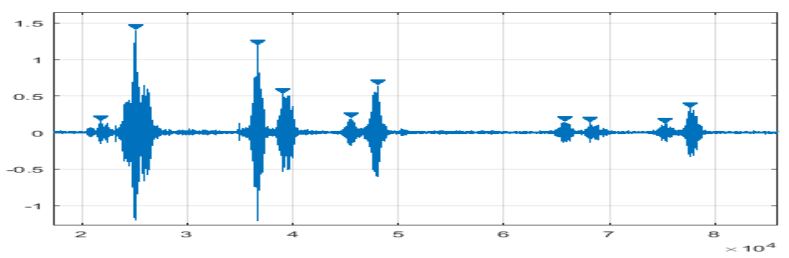

Fig. 5. Sum in time of all diferent take-off angles. 


\section{Simulation RESUlts}

In order to check the accuracy of each technique, we prepare 3 different simulations, which correspond to a 3 different scenarios, the first one, "Fig 6"; we simulate a wellknown environment without any variation at the received TDOA generated by mismatch between the model and real environment. This situation correspond the best case scenario, however it seems to be improbable due to some small fluctuations in SSP and imprecision at bottom depths. The second configuration, "Fig 7"; we simulate all path suffer a small variation in time. At each ray path we add a small random time interval, which correspond to variation of environmental parameters. The horizontal stratification of the oceans cannot be assumed at this case. This scenario corresponds to the worst possible case. The last configuration, "Fig 8"; corresponds to the some random well-known ray paths and some ray path plus a time variation interval. This scenario is the closest to the real environment since that in real case some path will be well defined for previous studies.

The simulation was done based on 200 realizations for each one of 9 different SNR, calculated as the mean of the signal divided by the mean of noise of the received signal.

The transmitted signal was a hyperbolic frequency modulation (chirp) with time duration of $100 \mu$ s and frequency range of $300 \mathrm{KHz}$ to $700 \mathrm{KHz}$. The medium was considered as homogeneous, with a flat bottom and a constant SSP. The target was motionless. The sea states considered was 3 . The transmitted signal was considered a known signal with the duration less than the next time arrival ray path to avoid the interference, which is a limited factor for the correlation technique.

The random time interval was limited at $60 \mu \mathrm{s}$, which in our case, considering the SSP at $1475 \mathrm{~m} / \mathrm{s}$, corresponds a mismatch maximum at around $8.9 \mathrm{~cm}$ (10\% bottom depth).

In all simulations 4 techniques were evaluated. Starting for maximum Hausdorff Distance, which selects the farthest value, the mean Hausdorff Distance, which calculates the average of all peaks, the correlation, which take in count the phase of the signal, and the last one the correlation considering the signals envelope, phase was disregarded.

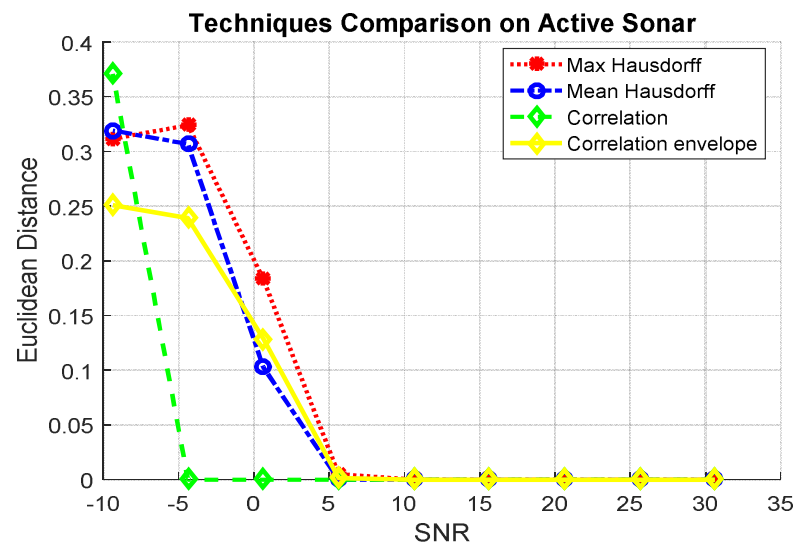

Fig. 6. First scenario - well-known enviroment, no variation in time.

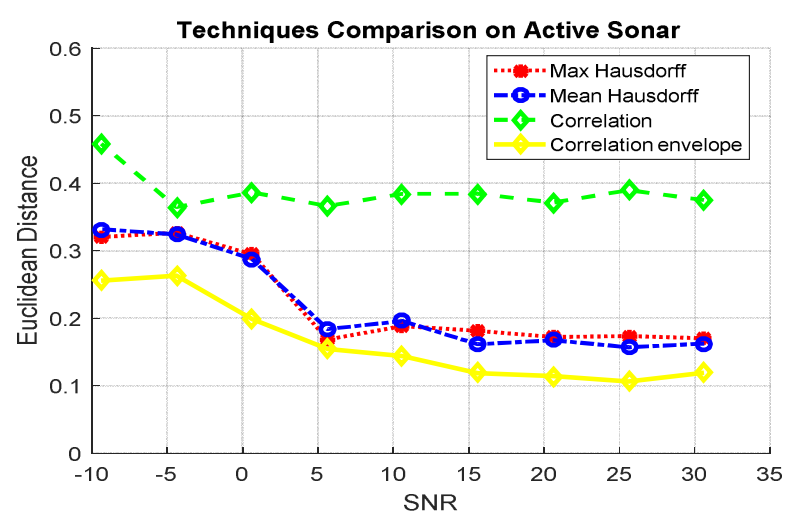

Fig. 7. Second scenario - all ray path suffer a small variation in time.

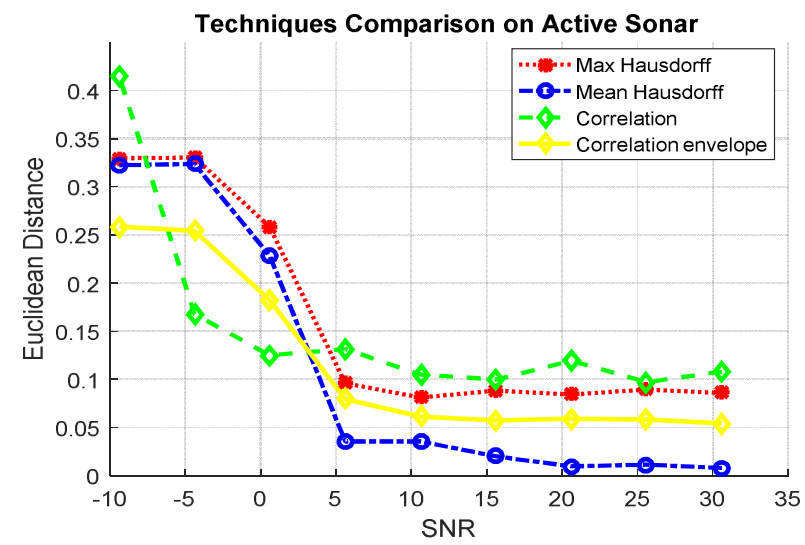

Fig. 8. Third scenario - random path suffer small variation in time.

In low SNR the correlation present better results and it can be explained due to some fake detection or false alarm after the matched filtering for the Hausdorff techniques. As we can see at "Fig 8", which is considered the closest to real environment the Mean Hausdorff Distance presents the best results because it can soften the path with larger discrepancy in time.

In terms of processing time the Hausdorff Distance takes $0.02 \mathrm{~s}$, the correlation in frequency domain $1.15 \mathrm{~s}$ and the correlation of the signal envelope in time domain $2.48 \mathrm{~s}$, which represents, using Hausdorff, an improvement around 58 and 124 times respectively.

\section{TECHNIQUES RESULTS}

Both Hausdorff techniques were used to evaluate the localization at the tank's experiment. The real target is located in the red line intersection. The dark blue means the minimum error that correspond the best result. The Mean Hausdorff Distance shows better resolution, which means small variance of error. In order to compare the most accurate technique, the results have been normalized and kept the same logarithmic scale being possible to direct compare the accuracy of each technique as shown the histogram at "Fig 7 and Fig 8".

This result shows us that we can localize a target using both techniques with good accuracy. The table 1 shows the results after the interpolation with a step of $0.005 \mathrm{~m}$ and the error 
when compared with the real position, $0.7 \mathrm{~m}$ range and $0.3 \mathrm{~m}$ deep.

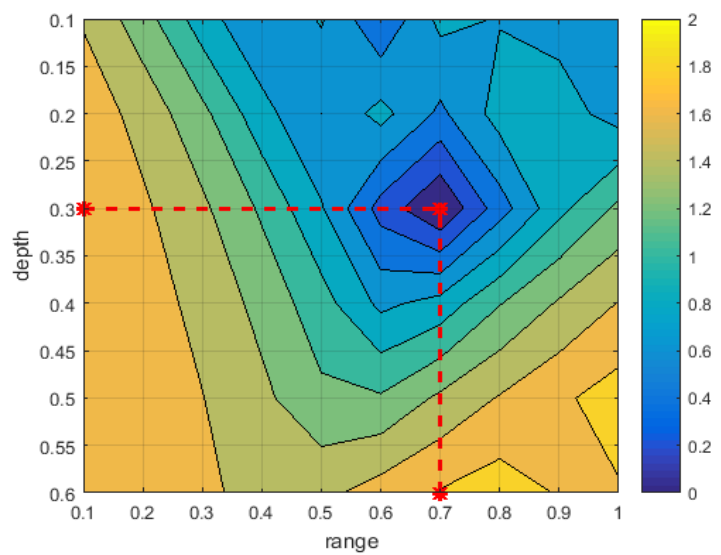

Fig. 9. Hausdorff Distance.

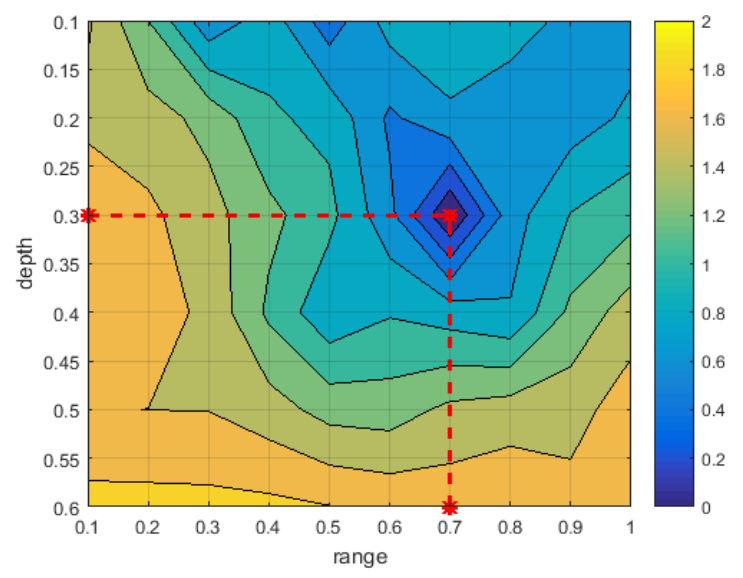

Fig. 10. Mean Hausdorff Distance.

TABLE I. LOCALIZATION ACCURACY

\begin{tabular}{|l|l|l|l|}
\hline & & $\begin{array}{l}\text { Max Hausdorff } \\
\text { Distance }\end{array}$ & $\begin{array}{l}\text { Mean Hausdorff } \\
\text { Distance }\end{array}$ \\
\hline \multirow{3}{*}{ Estimated } & Range & 0,705 & 0,69 \\
\cline { 2 - 4 } & Depth & 0,26 & 0,275 \\
\hline \multirow{3}{*}{ Error } & Range & 0,005 & 0,01 \\
\cline { 2 - 4 } & Depth & 0,04 & 0,025 \\
\hline \multirow{2}{*}{ Total Error } & 0,0403 & 0,0269 \\
\hline Area & 0,0011 & 0,0005 \\
\hline \multicolumn{2}{|l|}{ Total Error X Area } & $4,43 \mathrm{E}-05$ & $1,35 \mathrm{E}-05$ \\
\hline
\end{tabular}

\section{CONCLUSION}

We have proposed in this paper, innovative and efficient Hausdorff techniques to passive underwater localization. Overall the new technique demonstrates accuracy in range localization. As a theoretical feasibility, our investigation has shown that we can localize a target in a 2-D scenario, with a good precision, with no a prior information about source, using a passive approach with only one sensor at the receiver. As an experimental feasibility, we could localize the target in a tank experiment, with satisfactory results for all techniques. The 2 techniques utilized a priori for image localization, shown to be robust for underwater localization.

\section{REFERENCES}

[1] L. L. Brekhovskikh, Fundamentals of Ocean Acoustics.3th ed., New York: AIP PRESS, Springer., 2003.

[2] J. F. B. and W. A., Computational Acoustics, 2nd ed., New York: Springer., 2011.

[3] L. E. 3.Kinsler, Fundamentals of acoustics,4th ed., New York: John Wiley \& Sons, 2000

[4] N. E. Hurt, "Maximum likelihood estimation and MUSIC in array localization signal processing: A review," Multidim. Systems and Signal Processing, p. 279-325, 1989.

[5] Ozard, "Matched field processing in shallow water for range, depth, and bearing determination: Results of experiment and simulation," JASA, 1989 .

[6] Q. Zhang and J. Huang, "Joint estimation of DOA and time-delay in underwater localization", IEEE-ICASSP, Phoenix, 1999.

[7] P. Bustos, F. Donoso, A. Guesalaga and M. Torres, "Matching Radar and Satellite Images Employing the Hausdorff Distance for Ship Positioning and Trajectory Estimation," in 2006 Int. Conf. on Image Processing, Atlanta, 2006.

[8] M. Torres-Torrit and A. Guesalaga, "Automatic ship positioning and radar biases correction using the hausdorff distance," in 10th Int. Conf. on Information Fusion, Quebec, 2007.

[9] A. Andreev and N. Kirov, "Word Image Matching Based on Hausdorff Distances," in 10th Int. Conf. on Document Analysis and Recognition, 2009.

[10] S. H. Kim and R.-H. Park, "An efficient algorithm for video sequence matching using the modified Hausdorff distance and the directed divergence," IEEE Trans. on Circuits and Systems for Video Technology, pp. 592-596, 2002.

[11] H. Peyvandi, B. Fazaeefar and H. Amindavar, "Determining class of underwater vehicles in passive sonar using hidden Markov model with Hausdorff similarity measure," in Proceedings of 1998 Int. Conf. on Underwater Technology, Tokyo, 1998.

[12] T. L. Hemminger and Y.-H. Pao, "Detection and classification of underwater acoustic transients using neural networks," IEEE Trans. on Neural Networks, pp. 712-718, 1994.

[13] S. Reed, Y. Petillot and J. Bell, "Automated approach to classification of mine-like objects in sidescan sonar using highlight and shadow information," IEE Proceedings - Radar, Sonar and Navigation, pp. 48$56,2004$.

[14] A. Mours, C. Ioana, J. Mars, N. Josso and Y. Doisy, "Target-depth estimation in active sonar: Cramer-Rao bounds for a bilinear soundspeed profile," JASA, 2016.

[15] D. P. Huttenlocher, G. A. Klanderman and W. J. Rucklidge, "Comparing images using the Hausdorff distance," IEEE Trans. Pattern Analysis and Machine Intelligence, pp. 850-863, 1993.

[16] M. P. Dubuisson and A. K. Jain, "A modified Hausdorff distance for object matching," in Proceedings of 12th Int. Conf. on Pattern Recognition, Jerusalem, 1994.

[17] M. B. Porter, Y. Stephan, X. Demoulin, S. Jesus and E. Coelho, "Shallow-water tracking in the Sea of Nazare," in Proceedings of 1998 Int. Conf. Underwater Technology, Tokyo, 1998. 